Sagesse orientale « ad usum domesticum » :

Viazemski et Pouchkine

\title{
Andreï Dobritsyn
}

\section{(2) OpenEdition}

\section{Journals}

Édition électronique

URL : http://journals.openedition.org/edl/375

DOI : $10.4000 /$ edl. 375

ISSN : 2296-5084

\section{Éditeur}

Université de Lausanne

\section{Édition imprimée}

Date de publication : 15 septembre 2009

Pagination : 37-58

ISBN : 978-2-940331-20-8

ISSN : 0014-2026

\section{Référence électronique}

Andreï Dobritsyn, «Sagesse orientale " ad usum domesticum » : Viazemski et Pouchkine », Études de lettres [En ligne], 2-3| 2009, mis en ligne le 15 septembre 2012, consulté le 19 décembre 2020. URL : http://journals.openedition.org/edl/375 ; DOI : https://doi.org/10.4000/edl.375 


\section{SAGESSE ORIENTALE "AD USUM DOMESTICUM» : VIAZEMSKI ET POUCHKINE}

La première partie de l'article présente les sources occidentales des fables orientales de Viazemski, dont la majorité remonte aux apologues de Saadi. Il apparaît que les sujets de ces fables, bien connus en France au XVIII ${ }^{\mathrm{e}}$ siècle, ont eu un développement parallèle en France et en Russie au XIX ${ }^{\mathrm{e}}$ siècle. La deuxième partie de l'article retrace l'histoire d'une apophtègme de Saadi qui devient un motif récurrent chez Pouchkine.

\section{Fables persanes chez Viazemski}

On affirme ou on sous-entend souvent que «l'exotisme est [...] lié à ce thème fondamental de la littérature mondiale, le voyage. Sans départ, au moins imaginaire, pas de découverte ni de rêve concernant les horizons lointains " ${ }^{1}$. Il semble cependant que cette assertion ne concerne que les œuvres originales de la littérature occidentale, dans lesquelles la présence de l'exotisme doit être motivée. Or, le vrai goût de l'exotique littéraire, qui se développe dès le début du XVIII siècle, prend naissance essentiellement dans les traductions d'Antoine Galland ${ }^{2}$. Autrement dit, du point de vue de l'histoire littéraire, la source de la mode de l'exotisme se trouve dans les traductions et dans les imitations des textes orientaux ${ }^{3}$.

I. J.-M. Moura, Lire l'Exotisme, p. 3.

2. Sur la formation du genre du conte oriental en France voir l'article de J.-F. Perrin, "L'invention d'un genre littéraire au XVIII" siècle: le conte oriental».

3. Il va de soi que le degré de leur "exotisme" pouvait être amplifié ou atténué par un traducteur/imitateur. Cf. R. Robert, "Lecture croisée d'un conte oriental: Pétis de La Croix (Les Mille et Un Jours, 1710), $\mathrm{M}^{11 \mathrm{le}}$ Falques (Contes du sérail, 1753)», p. 39. 
Dans ces ouvrages, les éléments "exotiques" n’avaient évidemment pas besoin d'être justifiés par un motif de voyage.

Quand on étudie les œuvres traduites et imitées, il est plus utile de se rappeler les propos de Frank Lestringant:

L'exotique, au départ, est une chose, ou plutôt une liste de choses, un catalogue d'objets précieux - le butin des merveilles ramenées d'une campagne militaire, ou [...] d'un négoce sur les docks d'un grand port $[\ldots]^{4}$.

En effet, si la fable orientale n'est aucunement liée aux voyages (fussentils imaginaires), une œuvre étalant des apophtegmes de la sagesse orientale peut tenir lieu de "catalogue d'objets précieux».

La sagesse qui provenait de l'Orient était souvent considérée comme plus ancienne, donc plus "authentique», plus profonde, supérieure à la pensée occidentale, donc vraiment "précieuse». Ce n'est pas pour rien si au XVIII siècle on regardait parfois Loqman (le mythique fabuliste arabe) comme le prédécesseur (et de plusieurs siècles!) d’Esope.

Ce qui mériterait d'être étudié, c'est la figure du Sage oriental, par opposition à la figure du Savant occidental. "L'Asie a ses prophètes, l'Europe a ses docteurs", dit Edgar Quinet dans le Génie des religions, cité par Edward Said ${ }^{5}$.

Au Moyen Age, ce n'étaient pas les négociants occidentaux de retour de voyage, mais plutôt les Européens originaires de l'Orient qui mettaient à jour de pareilles perles de sagesse: il suffit de nommer Pierre d'Alphonse, créateur de la Disciplina clericalis (source de plusieurs fabliaux et nouvelles européens) et Jean de Capoue, traducteur de Kalilah et Dimnah (source de plusieurs fables européennes), deux juifs convertis dont les ancêtres avaient apporté en Europe leur bagage littéraire. La pensée orientale est venue en Europe d'elle-même.

Il faut avouer que vers les $\mathrm{XVI}^{\mathrm{e}}-\mathrm{XVII}{ }^{\mathrm{e}}$ siècles, la situation s'inverse: ce sont maintenant les Européens qui «rapportent le butin» intellectuel de leurs voyages en Orient. Quant aux Russes, ils ont importé (malgré leurs frontières communes avec les pays «exotiques») la sagesse orientale de l'Occident.

4. F. Lestringant, «L'Exotisme en France à la Renaissance», p. 11.

5. E. Said, L'Orientalisme: l'Orient créé par l'Occident, p. 97. 
Vers la deuxième moitié du XVIII ${ }^{\mathrm{e}}$ siècle le goût oriental à la française pénètre dans la littérature russe ${ }^{6}$; son influence est encore perceptible au début du XIX ${ }^{\mathrm{e}}$ siècle. Parmi les poètes du cercle pouchkinien, c'est Piotr Viazemski qui a laissé les exemples les plus caractéristiques de la fable orientale. Nous allons montrer que les sources de ses apologues se trouvent dans la poésie française, à l'exception d'une pièce, due au fabuliste polonais Ignacy Krasicki (qui, à son tour, s'appuyait sur une version française de l'apologue persan). Dans la plupart des cas, Viazemski a conservé le coloris oriental des pièces traduites; cependant, dans une seule épigramme il a jugé possible ou même nécessaire de le supprimer, pour pouvoir appliquer la thèse exprimée dans la pointe à la situation russe.

Dans ces notes nous allons citer les sources de six poésies de Viazemski. La plupart de ces sujets "orientaux" étaient très connus à l'époque et existaient en plusieurs versions; nous illustrerons par de nombreux exemples cette assimilation des «motifèmes» orientaux par les littératures française et russe.

\section{1. "Pour un portrait de Menchikov»}

En 1810 Viazemski a fait publier dans le Vestnik Evropy l'inscription "Pour un portrait de Menchikov»:

Kak volny, nam dary Fortuny nenadežny,

Sčastlivec, ne gordis'!

Carevy milosti s pogibeliju smežny!

Vzgljani na obraz sej... i sčastija strašis' ${ }^{7}$.

L'original est l'«Inscription sur la disgrâce de Giafer le Barmécide» composée par Voltaire et publiée dans plusieurs recueils de l'époque:

Mortel, foible mortel, à qui le sort prospere

Fait goûter de ses dons les charmes dangereux;

6. V. N. Kubačeva, «'Vostočnaja povest' v russkoj literature XVIII - načala XIX veka».

7. P. A. Viazemskij, Stihotvorenija, p. 51; Polnoe sobranie sočinenij, p. 14. 
Connois quelle est des rois la fâveur passagere,

Contemple Barmécide, \& tremble d'être heureux ${ }^{8}$.

Cette «Inscription» contient une allusion à l'histoire de l'essor et de la chute des Barmécides pendant le règne de Harun ar-Rachid, racontée dans les chroniques de Tabari. Voltaire pouvait trouver cette histoire dans l'édition de Gulistan de Saadi, faite en 1704 par d'Alegre. Dans une annexe intitulée "Augmentations aux Rois et Kaliphes, de Saadi, Tirées des Auteurs Arabes, Persans, et Turcs», on voit un quatrain exposé en prose:

Nourrisson de la fortune, qui succez pendant quelque jours le laict de la prospérité qui coule de ses mamelles empoisonnées, ne te vante pas trop du bonheur de ton état pendant que tu es encore dans le berceau suspendu d'une vie toujours en mouvement.

Souviens-toi du tems où tu as vû la grandeur des Barmecides?.

Viazemski a plusieurs fois trouvé de nouvelles applications aux épigrammes françaises, en les adressant heureusement à ses contemporains russes. On pourrait citer ses attaques contre $S$. Bobrov (d'après Voltaire), contre D. Khvostov (d'après Rulhière), et autres. L'inscription "Pour un portrait de Menchikov» est adaptée à l'histoire russe du XVIII ${ }^{\mathrm{e}}$ siècle, le coloris oriental de l'original s'est effacé au cours des transformations consécutives: d'abord, c'est Voltaire qui a supprimé les images et les tropes jugés trop «orientaux» et contraires au "bon goût", ensuite Viazemski a éliminé le nom propre, dernière trace de l'exotique original ${ }^{10}$.

8. Elite de Poësies fugitives, t. 4, p. 5; Almanach des Muses, p. 32; Les Fléches d'Apollon ou Nouveau Recueil d'Epigrammes anciennes et modernes, t. 2, p. 27; Petite Encyclopédie poétique ou Choix de Poésies dans tous les genres, t. 3, p. 194.

9. M. Saadi, Gulistan ou l'Empire des Roses, Traité des Mours de Rois, composé par Musladini Saadi, Prince des Poetes Persiens, p. 227-228.

Io. Remarquons le changement de la signification normale du mot sčastije «bonheur ": dans l'épigramme citée (comme dans plusieurs autres poèmes russes traduits du français) il signifie «fortune» (cf. «slučaj» dans le sens de la "grâce passagère royale»). 


\section{2. «La Goutte»}

La fable «La Goutte» (dans la table des matières elle portait le sous-titre «Apologue oriental») a été publiée en 1823 dans les Novosti literatury:

S lazurnyh niv upala kaplja v more.

K komu pristat' sirotke bednoj gore!

No na nee vzgljanul dnja svetlyj bog

I vzgljad ego byl ej nadežd zalog,

Ee sokryl on v rakovine skudnoj

I s kapleju sveršilsja žrebij čudnyj:

Bogatyj perl, doždavšis' lučših dnej,

Blestit ona v vence Carja-carej!

Umej terpet'! Pridet nagrady vremja :

V smirenii velič ja zreet semja ${ }^{11}$.

La fable remonte à l'apologue qui se trouve dans le Boustan de Saadi. Cette œuvre était beaucoup moins connue en France que Gulistan: la première imitation, libre et fragmentaire, n'a paru qu'en 1762, et la première traduction véritable qu'en 1869. Cependant, l'apologue de la goutte n'était pas inconnu du public. Au début du XVIII e siècle déjà, il était exposé dans la traduction du Spectateur anglais ${ }^{12}$. En 1766 le même apologue était traduit de la version anonyme allemande qui venait d'être publiée $^{13}$. Un orientaliste réputé, Louis Mathieu Langlès (1763-1824) donnait encore une traduction:

Une Goutte d'Eau tomba des nuages et fut couverte de confusion en contemplant la vaste étendue des mers. "Où suis-je, dit-elle? Qu'est-ce que la mer? Et que suis-je moi-même? Si tout cela existe, est-il bien vrai que j'existe aussi?» Tandis qu'elle s'examinait avec dédain, une coquille à perle la prit et la conserva. Le destin voulut que cette faible goute d'Eau devint le plus précieux joyau des Rois. Elle trouva son élévation dans son abaissement, et elle passa du néant à la lumière ${ }^{14}$.

II. P. A. Viazemskij, Polnoe sobranie sočinenij, t. 3, p. 201.

I2. Le Spectateur ou le Socrate moderne. Où l'on voit un Portrait naïf des Mours de ce Siècle, p. 305-306.

13. M. Huber, Choix de Poésies Allemandes, t. 2, p. 317-318.

I4. L. M. Langlès, Contes, Fables et Sentences, Tirés de différens auteurs arabes et persans, avec une analyse du poëme de Ferdoussy, sur les Rois de Perse, p. 36. 
A la fin du XVIII e siècle parut encore une traduction du persan ${ }^{15}$. La bibliographie de Saadi n'indique que la version de Langlès ${ }^{16}$.

Jan Potocki dans son Voyage en Turquie et en Egypte, conte l'histoire d'un homme qui obtient la faveur du sultan grâce au même apologue:

Une goutte d'eau échappée à la nue, tomba un jour dans la mer. Effrayée d'abord de l'immensité de l'élément dans lequel le sort l'avoit jetée, elle perdit l'usage de ses facultés; mais une coquille la reçut dans son sein, la nourrit, la protégea, \& cette goutte d'eau est devenue dans la suite la perle qui orne le diadême de ta Hautesse ${ }^{17}$.

Mentionnons une version plus ample (25 vers) de Mancini-Nivernois, dont «La Goutte d'eau "s'éloigne significativement du prototype ${ }^{18}$. En revanche, «La Goutte d'Eau» d'Antoine Pierre Dutramblay (1745-1819) reste relativement laconique:

Au fond des mers s'engloutissant, La Goutte d'Eau disait: je ne suis rien au monde!

Souverain directeur de la machine ronde,

Pourquoi me sortir du néant?

Dans ce moment

Une huître baille:

Au beau milieu de son écaille

Elle reçoit la Goutte d'Eau,

Qui s’y durcit, et devient perle fine.

I5. «Une goutte de pluie s'écoulant d'un nuage tomba un jour dans la mer. Honteuse et confuse en se voyant dans cet espace immense, elle dit: Que suis-je en comparaison de ce vaste océan, certes mon existence est moins que rien au milieu de cet abyme sans bornes. Tandis qu'elle parlait d'elle-même avec tant de dédain et de mépris, une coquille de nacre la reçut dans son sein, et la fortune l'y favorisa tellement, qu'elle devint une perle précieuse et magnifique, digne d'orner la tête des rois: ainsi elle trouva dans son abaissement même la cause de son élévation, et la source de son illustration fut l'obscurité même où elle avait été plongée»; Lukman, Fables de Loqman, surnommé le Sage. Edition Arabe, accompagnée d'une Traduction française, et précédée d'une Notice sur ce célèbre Fabuliste, p. 20.

I6. H. Massé, Essai sur le poète Saadi, suivi d'une bibliographie.

17. J. Potocki, Euvres [t.] 1: Voyage en Turquie et en Egypte. Voyage en Hollande. Voyage dans l'Empire de Maroc, suivi du Voyage de Hafez. Voyage dans quelques parties de la Basse-Saxe, p. 33.

I8. L. J. Mancini-Nivernois, Fables, t. 1, p. 63-64. 
Le Ciel tire souvent ce qu'on voit de plus beau,

De la plus obscure origine ${ }^{19}$.

Un motif manque chez Dutrambley: dans sa version, la goutte, transformée en perle, n'orne pas de couronne.

Auguste Creuzé de Lesser (1771-1839) a tiré de la même histoire une morale complètement différente: il termine son apologue «La Goutte d'eau» en appelant le lecteur à trouver sa propre huître:

Une goutte d'eau, de la nue,

Tombe en l'océan irrité,

Et croyait bien, faible, inconnue,

Périr dans son immensité.

Dans une coquille entr'ouverte,

Voilà qu'elle échappe à sa perte,

S'y consolide lentement,

Et bientôt, perle ravissante,

S'en va d'une tête charmante

Etre le plus bel ornement.

Ainsi souvent s'élève une grande famille.

Ne désespérez pas dans les moindres emplois.

On devient perle quelquefois;

Mais il faut trouver sa coquille ${ }^{20}$.

Il est très probable que la plupart des auteurs mentionnés ont pris connaissance de ce sujet grâce au père François-Joseph Terrasse Desbillons (ou Des Billons, 1711-1789), dont les fables latines jouissaient d'une certaine popularité; en 1779 et en 1809, elles ont été éditées avec la traduction française en regard. L'apologue «Gutta, Pontus et Ostreum» (IX.12) est assez proche du texte original de Saadi ${ }^{21}$.

Viazemski, ainsi que plusieurs traducteurs et imitateurs français de Saadi, a préféré garder une certaine "orientalité» de la fable, mais les versions citées plus haut nous montrent que les connotations orientales peuvent aussi être facilement éliminées.

19. A. P. Dutramblay, Apologues, p. 22.

20. A. Creuzé de Lesser, Apologues, p. 24.

2I. F.-J. Desbillons, Fables du Père Desbillons Traduites en François, Avec le texte latin Corrigé de nouveau par l'Auteur, t. 2, p. 214-217. 
3. «La colère d'un vertueux: Apologue oriental»

La fable «La colère d'un vertueux: Apologue oriental» fut publiée dans le Slavjanin en 1827:

Car' dobrodetel'nyj, no vspyl'čivyj i dikoj,

Nevinnomu rabu glavu velel otseč' :

"Ne strašen dlja menja blestjaščij smert' ju meč" », -

$S$ rešimost' ju skazal nevol'nik pred vladykoj

«Menja zlosčastnee moj ljutyj sudija;

«Ty s žizn’ju mne prerveš mgnovennoj kazni muku,

N s smertiju moej načnetsja kazn’ tvoja!» -

I Car' k nemu proster spasitel'nuju ruku ${ }^{22}$.

L'original de cet apologue, intitulé "Despote», se trouve dans les Fables orientales de Saint-Lambert:

Un Roi vertueux, dans un moment de colère, alloit faire périr un innocent. O Roi, lui dit-il, mon supplice va finir avec ma vie; mais le tient va commencer. Le Roi fit grace ${ }^{23}$.

Creuzé de Lesser l'a mis en vers dans le quatrain «L'Innocent sauvé»:

Un innocent prêt à périr,

Du sultan bravant la menace,

Dit: Mon supplice va finir,

Le tien commence. Il eut sa grace ${ }^{24}$.

Une version modifiée et beaucoup plus prolixe (en sept alexandrins) a été proposée par A.-E.-X. Poisson de La Chabeaussière ${ }^{25}$.

L'histoire est tirée du premier chapitre du Boustan de Saadi. Le cruel Haddjadj avait exécuté un innocent; la nuit suivante un "pieux personnage» vit en songe le supplicié qui lui dit: "Mon supplice n’a duré qu’un

22. P. A. Viazemskij, Polnoe sobranie sočinenij, p. 435.

23. J.-F. de Saint-Lambert, Les Saisons, Poëme, Euvres diverses de l'Auteur des Saisons, p. 334 .

24. A. Creuzé de Lesser, Apologues, p. 117.

25. A.-E.-X. La Chabeaussière, Apologues moraux, imités pour la plupart de Saadi le Persan, p. 7. 
instant, mais celui qui l'a ordonné en subira le châtiment jusqu'au jour du jugement ${ }^{26}$.

\section{4. «Le Derviche et son Disciple»}

La fable "Le Derviche et son Disciple» parut en 1819 dans le Fils de la Patrie (Syn Otečestva):

Učenyj muž, Dervis, v čas utra i obeda

Svjatuju vodu pil v kolodce Magomeda.

Podmetil to ego nelepyj učenik,

I na den' begal pit' raz dvadcat' na rodnik.

Kakoj že on imel uspeh s svoej dogadki?

Ostalsja neučen i sleg ot lihoradki ${ }^{27}$.

C'est une traduction de la fable «Derwisz i Uczeń» d'Ignacy Krasicki ${ }^{28}$. Le poète polonais imita probablement un des «Apologues orientaux" de Louis-Edme Billardon de Sauvigny (1736 ?-1812), intitulé «Le Disciple qui veut surpasser son Maître»:

Abdalla, l'homme le plus sçavant de son siècle \& le plus laborieux, attribuoit sa sçience à l'eau du puit de la Mecque, qu'il buvoit avec une très-grande dévotion; un de ses Disciples, croyant faire mieux que lui, renonça aux livres, \& ne s'occupa qu'à boire l'eau du puits sacré; il vouloit devenir sçavant, il devint hydropique ${ }^{29}$.

Cet apologue fut mis en vers par Creuzé de Lesser, sous le titre «Le Maître et l'écolier»:

A l'eau du puits sacré je dois tout mon savoir,

Disait un musulman, des lois sage interprète.

Il en buvait souvent, et, malgré son pouvoir,

Par l'étude, matin et soir,

Il secondait les bontés du prophète.

26. M. Saadi, Le Boustan ou Verger, Poème persan de Saadi, p. 53.

27. P. A. Viazemskij, Polnoe sobranie sočinenij, p. 173.

28. S. I. Nikolaev, Ot Kohanovskogo do Mickeviča. Razyskanija po istorii pol'skorusskih literaturnyh svjazej XVII - pervoj treti XIX v., p. 221.

29. L.-E. Billardon de Sauvigny, Apologues orientaux, Dédiés à Monseigneur Le Dauphin, p. 19. 
Un jeune homme charmé d'un exemple si beau

Le comprit assez mal, bien qu'assez il s'explique:

Il n'étudia plus, but toujours de cette eau,

Crut devenir savant, et devint hydropique ${ }^{30}$.

Par une curieuse coïncidence, le poème suivant, qui remonte également à Billardon de Sauvigny, a lui aussi été imité par Viazemski ainsi que par Creuzé de Lesser.

5. «Les Dents et la Langue: Apologue oriental»

La fable «Les Dents et la Langue: Apologue oriental» fut publiée dans les Novosti literatury en 1823 :

Odin sultan penjal sedomu vizirju,

Čto tverdoj stojkosti on ne imel vo nrave.

«Za nedostatok sej sud'bu blagodarju!

Im uderžalsja ja i v počesti i v slave, -

Skazal vizir' emu, — i pri dvore tvoem

Sred' častyh peremen on byl moim ščitom.

Mne šest'desjat pjat' let, — pribavil on s ulybkoj, -

Iz tverdyh teh zubov, kotorye imel,

Ty vidiš - redkij ucelel;

No vse ih perežil odin jazyk moj gibkoj» ${ }^{31}$.

Tout comme la fable précédente, ce dixain a deux parallèles français: un apologue prosaïque de Sauvigny et une pièce versifiée de Creuzé de Lesser. Voici le premier («La Langue \& les Dents»):

Un Sultan reprochoit à un de ses Visirs de n'être pas d'un caractere assez ferme: Sire, lui répond le Visir; c'est à ce même caractere que j'ai l'obligation de la place que j'occupe auprès de Ta Hautesse; \& c'est par lui que je m'y maintiens. Il y a soixante ans que je suis au monde; mes dents, qui étoient dures, sont presque toutes tombées; ma langue, qui ne l'étoit pas, m'est restée toute entiere ${ }^{32}$.

30. A. Creuzé de Lesser, Apologues, p. 37.

31. P. A. Viazemskij, Stihotvorenija, p. 156-157; Polnoe sobranie sočinenij, p. 317.

32. L.-E. Billardon de Sauvigny, Apologues orientaux, Dédiés à Monseigneur Le Dauphin, p. 153. 
Nous pouvons comparer ce texte avec la version versifiée ( Les Dents et la Langue»):
Il faut avoir du caractère,
Mais il n'en faut pas trop pourtant.
Voici ce qu'en Bagdad un père
Disait un jour à son enfant,
Dont rien ne pliait l'humeur fière:
Mon ami, voilà soixante ans
Que j'ai commencé ma carrière;
Mes dents ont péri dès long-temps,
Et ma langue est encore entière ${ }^{33}$.

Deux micromotifs communs à Viazemski et Sauvigny, manquent chez Creuzé: 1) le sultan adresse un reproche à son vizir, 2) le vizir explique, comment il a su s'élever et garder son statut. La fable de Creuzé enseigne que la flexibilité est parfois plus utile dans la vie que la fermeté (cf. «Le Chêne et le Roseau" et ses nombreuses versions). En revanche, dans les apologues de Sauvigny et de Viazemski la morale est ironique: l'apologie de la flexibilité est faite par un courtisan flatteur qui se conforme toujours à l'opinion du souverain.

Il y a au moins encore une version de la même histoire: "Le Vieux Vizir. Apologue» de l'abbé de Schosne, publié dans L'Almanach des Muses en $1792^{34}$. Dans ce huitain ce n'est pas le sultan, mais les amis du vizir qui lui reprochent son conformisme.

6. «Le Calife et Le Derviche: Apologue oriental»

«Le Calife et Le Derviche: Apologue oriental» fut publié par Viazemski dans la Revue des Dames (Damskij Žurnal) en 1823. Cette œuvre se situe entre la fable et le conte en vers; voici son sujet: un calife hautain, entouré de sa suite, rencontre près de ruines un vieillard qui tient un crâne dans ses mains. A une question du calife, le derviche répond qu'il essaie de deviner si le crâne appartenait à un roi ou à un mendiant -

33. A. Creuzé de Lesser, Apologues, p. 29.

34. Almanach des Muses, p. 110. 
tâche qui se révèle impossible. Suite à cette rencontre, le calife perd en partie son arrogance et se souvient qu'il n'est qu'un homme ${ }^{35}$.

Il est assez probable que Viazemski ait été inspiré par la fable de Mancini-Nivernois "Le Visir et le Manant» ${ }^{36}$; avec cela, il a emprunté le titre d'une autre fable de Mancini ("Le Derviche et le Calife»). La même histoire fut remaniée par Creuzé de Lesser ${ }^{37}$. Si certains détails (comme la description de la suite du calife) rapprochent Viazemski de Mancini, la morale de sa fable est plus proche de celle de Creuzé. Il n'est donc pas exclu que le poète russe ait "croisé» les deux textes français.

Il est intéressant de noter que quatre des six poèmes "orientaux" cités sont liés au thème du despote. L'un d'eux (l'inscription pour un portrait de Barmécide resp. de Menchikov) est tiré d'une véritable histoire orientale («le sort», qui a fait chuter les Barmécides, c’était la volonté du despote: le calife Harun ar-Rachid). Evidemment, une telle proportion n'est pas due au hasard: dans la description par Hérodote des guerres gréco-persanes, l'Orient est déjà associé au règne de l'arbitraire, à l'idée d'un gouvernement tyrannique (au sens moderne du mot). L'histoire du terme "despotisme» (et "despotisme oriental» en particulier) est méticuleusement étudiée par Koebner ${ }^{38}$ et Venturi ${ }^{39}$. Au XVIII ${ }^{\mathrm{e}}$ siècle, l'usage de ce terme était assez courant; chez certains représentants de la philosophie politique des Lumières, il jouait un rôle important, comme chez Montesquieu ou comme dans les fameuses Recherches sur l'origine $d u$ despotisme oriental de Nicolas-Antoine Boulanger (1761, nombreuses rééditions ${ }^{40}$. Dans ce genre si conventionnel qu'est la fable, il est naturel que le motif du despotisme soit régulièrement habillé d'un «discours oriental» (lui aussi conventionnel).

Remarquons également une évolution parallèle de certains thèmes propres aux fables françaises et russes: les mêmes sujets, exploités en France au XVIII e siècle, sont jugés dignes d'être réexplorés en France et en Russie au XIX ${ }^{\mathrm{e}}$ siècle.

35. P. A. Viazemskij, Polnoe sobranie sočinenij, p. 324-325.

36. L. J. Mancini-Nivernois, Fables, t. 1, p. 73-75.

37. A. Creuzé de Lesser, Apologues, p. 93-94, «La Tête».

38. R. Koebner, "Despot and Despotisme: Vicissitude of a Political Term».

39. F. Venturi, Europe des Lumières. Recherches sur le $18^{e}$ siècle.

40. Les Recherches sur l'origine du despotisme oriental étaient aussi attribuées à PaulHenri Thiry D'Holbach, à Denis Diderot et à d'autres auteurs. 
II. "Et ne dispute pas avec les ignorants»: Une apophtegme orientale chez Pouchkine

Le "Monument» de Pouchkine (1836, Je me suis érigé un monument..., libre imitation de "Exegi monumentum..." horacien) fut l'objet de nombreux commentaires et d'une monographie écrite par un des meilleurs spécialistes soviétiques ${ }^{41}$. En particulier, on a discuté à plusieurs reprises le vers final du poème pouchkinien, qui n'a pas d'équivalent dans l'ode 3.30 d'Horace: «Et ne conteste pas avec le sot». Wilhelm Busch a remarqué qu'une pensée semblable se trouvait dans une autre ode horacienne: la $2.16^{42}$, mais un parallèle plus proche existe chez Pouchkine lui-même: M. P. Alekseev rappelle les vers de «L'Album d'Onéguine» ${ }^{43}$ :

V Korane mnogo myslej zdravyh, Il y a beaucoup de pensées sensées dans le Coran,

Vot naprimer: pred každym snom Molis' — begi putej lukavyh Čti boga i ne spor's glupcom — ${ }^{44}$ Par exemple: avant de dormir Prie, évite les voies malignes, Vénère Dieu et ne discute pas avec le sot.

Pouchkine essayait également d'autres variantes: "Aime les orphelins, fuis les malins, [fuis] les pensées malignes, Ne discute pas avec l'ignorant et le sot, etc. ${ }^{45}$.

Il semble que l'on puisse indiquer la source directe de ces paroles: un quatrain de Louis-François Dubois (ou Du Bois, 1773-1855), publié dans L'Almanach des Muses en 1807 et intitulé "Conseils de Mahomet, Tirés du Coran »:

Chérissez qui vous hait, donnez à qui vous ôte;

Envers tous les mortels montrez-vous bienfaisants;

4I. M. P. Alekseev, Stihotvorenie Puškina "Ja pamjatnik sebe vozdvig... ». Problemy ego izučenija.

42. W. Busch, Horaz in Russland, p. 48.

43. M. P. Alekseev, Stihotvorenie Puškina "Ja pamjatnik sebe vozdvig... ». Problemy ego izučenija, p. 223, n. 10.

44. A. S. Puškin, Polnoe sobranie sočinenij, t. 6, p. 614.

45. Ibid., p. 431 et 432 avec n. 1 et 2. 
Un méchant vous blessa, pardonnez-lui sa faute;

Et ne disputez pas avec les ignorants ${ }^{46}$.

Des cinq préceptes du quatrain français, seul le dernier trouve son équivalent dans "l'Album d'Onéguine" et dans le "Monument". Remarquons que c'est justement le vers qui, pour l'oreille russe, sonne de la manière la plus frappante, avec son rythme quasi syllabo-tonique (plus exactement, iambique).

Ces "Conseils" ont un parallèle allemand: le quatrain de Johann Wilhelm Ludewig Gleim (1719-1803), présenté comme une sentence de Mahomet (ce quatrain est précédé, en guise de titre, par les paroles "Mahomet, im Koran, sagte»):

Dem Menschen, welcher dich beleidigte, vergib;

Hab' alle Menschen lieb,

Und bringe frevelnd nicht aus seiner Seelenruh

Den Mann, der minder weis' wie du! ${ }^{47}$

Nous ne savons pas s'il existe un lien direct entre ces deux quatrains, mais de toute façon, ils remontent au recueil Les Paroles remarquables, les Bons mots et les Maximes des Orientaux composé par Antoine Galland et paru en $1694^{48}$. Galland ne cite pas ses sources, mais on voit facilement que la majorité des histoires, des apologues et des aphorismes est tirée du Gulistan de Saadi. Dans la deuxième partie du recueil (où sont réunies les «maximes des Orientaux»), nous trouvons le passage suivant:

46. Almanach des Muses, p. 142.

47. J. W. L. Gleim, Sämmtliche Werke. Erste Originalausgabe aus des Dichters Handschriften durch W. Körte, p. 313.

48. On peut consulter également une édition moderne d'A. Meddeb. Cf. A. Galland, Les Paroles remarquables, les bons mots et les maximes des Orientaux. Traduction de leurs ouvrages en arabe, en persan et en turc, avec des remarques. Antoine Galland est connu avant tout comme le traducteur (et en partie, le créateur) des contes des Mille et une nuits, mais Les Paroles remarquables... des Orientaux ont également influencé la littérature postérieure, y compris la littérature russe: plus d'une douzaine d'histoires tirées de ce recueil ont été traduites dans N. Kurganov, Pismovnik, soderžaščij v sebe nauku rossijskago jazyka, so mnogim prisovokupleniem raznago učebnago i poleznozabavnago vešceslovija, V. D. Rak, Russkie literaturnye sborniki i periodičeskie izdanija vtoroj poloviny XVIII veka, p. 293; A. A. Dobritsyn, "I ne osporivaj glupca...». 
Aiés le cœur pur \& net devant Dieu. Soyes generalement civil envers tout le monde. Maistrisés vos passions, soyes soumis à vos superieurs, $\&$ supportés leurs defauts. Prenés conseil des sages. Soyés doux envers vos ennemis, respectueux envers les Savans \& dans le silence devant les ignorans ${ }^{49}$.

Le conseil final de ce passage remonte, semble-t-il, à Saadi. Dans le quatrième chapitre du Gulistan ("Sur les avantages du silence») nous lisons:

Une contestation s'engagea entre un savant considéré et un ismaëlien. Le premier ne l'emporta pas sur le second par ses arguments. Alors il renonça à la dispute et s'en retourna [...]

Vers. - «Cette personne dont tu ne te délivres pas avec le Coran et la tradition, la réponse à lui faire, c'est de ne pas lui en faire. ${ }^{50}$

Du Ryer a traduit le dernier vers ainsi : «[...] la respóse qu’il faut faire à telle gens, c'est qu'il ne leur faut point faire de response " ${ }^{51}$. De plus, le dernier chapitre du Gulistan (VIII, "Touchant les bienséances de la société») contient une maxime, que Du Ryer a compris de la façon suivante: «Sois muet avec les ignorants, que si l'ignorant cognoissoit son ignorance, il ne seroit plus ignorant " ${ }^{52}$. C'est probablement à partir de

49. [A. Galland], Les Paroles remarquables, les Bons mots et les Maximes des Orientaux. Traduction de leurs Ouvrages en Arabe, en Persan et en Turc, avec des Remarques, p. 206; A. Galland, Les Paroles remarquables, les bons mots et les maximes des Orientaux. Traduction de leurs ouvrages en arabe, en persan et en turc, avec des remarques, p. 127.

50. M. Saadi, Gulistan ou le Parterre des Roses, p. 207-208.

5I. M. Saadi, Gulistan ou L'Empire des Roses, Composé par Sadi, Prince des Poëtes Turcs et Persans, p. 133.

52. Ibid., p. 172. La recommandation d'éviter la société des ignorants se trouve dans le Pend-Nameh de Saadi, dont le chapitre VIII commence ainsi : "Si tu es prudent et sage, ne fréquente pas l'ignorant; fuis loin de lui comme la flèche" (Exposition de la Foi Musulmane, p. 112). Dans une version anglaise de Francis Gladwin: "Choose not the society of ignorant" (FPL 1805, 123). Le Pend-Nameh traduit par Joseph Héliodore Garcin de Tassy, parut en 1822 en tant que troisième volume (complémentaire) de l'édition du Coran (dont les deux premiers volumes furent donnés dans la traduction de C. Savary, Le Coran, Traduit de l'Arabe, Accompagné de Notes, et précédé d'un Abrégé De la Vie de Mahomet, Tiré des Ecrivains Orientaux les plus estimés). En 1828, les trois volumes furent réimprimés (Exposition de la Foi Musulmane) et c'est cette édition qui fut acquise par Pouchkine. Cependant, il ne découpa que le «Pend-Nameh» (Modzalevskij 1910, p. 291-292). Il faut mentionner encore un Pend-Nameh de Farid ad-Din Attar, traduit en 1819; le chapitre LXXII recommande également d'éviter la mauvaise 
cette traduction que le précepte en question a pénétré dans la «section orientale» de la tradition aphoristique française (cf. par exemple, les «Pensées» en annexe aux Fables orientales de Saint-Lambert ${ }^{53}$ ).

Il semble que ces recommandations de Saadi aient également influencé le troisième poème du cycle pouchkinien "Imitations du Coran». Ce poème reprend le contenu de la $80^{\mathrm{e}}$ sourate, dont le début est cependant traité d'une manière très éloignée de l'original:

Smutjas', nahmurilsja prorok, Slepca poslyšav približen'e: Bežit, da ne derznet porok Emu javljat' nedoumen'e.

$S$ nebesnoj knigi spisok dan

Tebe, prorok, ne dlja stroptivyh; Spokojno vozveščaj Koran, Ne ponuždaja nečestivyh! ${ }^{54}$
Troublé, le prophète fronce les sourcils, En entendant s'approcher un aveugle: Il fuit, pour que le vice n'ose pas Lui montrer son incompréhension.

La copie du livre céleste ne t'est pas donnée, $\mathrm{O}$ prophète, pour les récalcitrants; Annonce tranquillement le Coran, Sans forcer les impies!

N. O. Lerner a déjà remarqué le caractère peu fidèle de l'interprétation pouchkinienne ${ }^{55}$. Citons les premiers versets de la $80^{\mathrm{e}}$ sourate dans la traduction de Savary:

Le prophète a montré un front sévère et a détourné les yeux, Parce qu'un aveugle s'est présenté devant lui;

Et qui pouvait t’assurer [diras-tu] ${ }^{56}$ qu'il était juste,

Ou qu'en l'avertissant tes conseils lui seraient salutaires?

Mais le riche qui nage dans l'abondance,

Tu le reçois avec distinction;

Il t'importe peu s'il est équitable.

société (A. I. Silvestre de Sacy, Pend-Namèh, ou Le Livre des Conseils de Férid-Eddin Attar, p. 291). Un autre parallèle se trouve dans les Mélanges... de Denis Dominique Cardonne: "La société de l'Ignorant est dangereuse pour le Savant» (D. D. Cardonne, Mélanges de Littérature Orientale. Traduits de différens Manuscrits Turcs, Arabes \& Persans de la Bibliothèque du Roi, p. 281).

53. J.-F. De Saint-Lambert, Fables Orientales de M. de S. Lambert, Auteur des Saisons, p. 14,44 et 62 .

54. A. S. Puškin, Polnoe sobranie sočinenij, t. 2/1, p. 353.

55. N. O. Lerner, "Primečanija. Stihotvorenija 1824 g.», p. 541.

56. La conjecture "diras-tu» est ajoutée dans une note en bas de page. 
Et celui que le zèle t'amène,

Qui craint le Seigneur,

Tu détournes de lui tes regards.

Cette conduite est injuste; que ce chapitre t'instruise ${ }^{57}$.

Savary cite encore un commentaire d'un savant exégète musulman:

Un aveugle se présenta devant Mahomet dans un moment où il travaillait à la conversion d'un Coréishite riche et puissant; il fut mal reçu. L'aveugle revint, et il fut traité avec bonté. Gélaleddinn ${ }^{58}$.

Ainsi, les paroles par lesquelles Allah s'adresse au prophète signifient: «tu te justifies en avançant que pour l'aveugle ton sermon serait inutile, mais en réalité, tu ne veux pas dépenser ton temps avec un pauvre, car tu t'es occupé avec un riche "59. Cependant, Pouchkine a compris ce texte dans l'esprit des conseils de Saadi, qui incitent à ne pas discuter avec les ignorants et les sots. Cela s'explique facilement: le poète russe a lu le Coran dans la traduction russe de Verevkine, faite à partir de la traduction française d'André Du Ryer ${ }^{60}$, où le fragment en question se présente ainsi :

Le Prophete a froncé le front, a eu le visage refrogné, \& s'est retiré lors que l'Aveugle est venu vers lui. Il ne te dira pas s'il croira en Dieu; \& s'il profitera de tes prédications; éloigne-toi de celui qui s'éloignera de la loi de Dieu, tu n'es obligé qu'à le prêcher, \& non pas à le faire croire [...] [cf. chez Pouchkine: Spokojno vozveščaj Koran, // Ne ponuždaja nečestivyh! = Annonce tranquillement le Coran, // Ne forçant pas les impies!] [... ${ }^{61}$.

57. C. Savary, Le Coran, Traduit de l'Arabe, Accompagné de notes, Précédé d'un abrégé De la Vie de Mahomet, Tiré Des écrivains orientaux les plus estimés, p. 369-370; Le Coran, Traduit de l'Arabe, Accompagné de Notes, précédé d'un Abrégé De la Vie de Mahomet, Tiré Des Ecrivains Orientaux les plus estimés, p. 412.

58. Ibid., n. 1.

59. N. O. Lerner, «Primečanija. Stihotvorenija 1824 g.», p. 541.

6o. K. S. Kaštaleva, "Podražanija Koranu” Puškina i ih pervoistočnik».

6I. A. Du Ryer, L'Alcoran de Mahomet. Traduit de l'Arabe, Par André du Ryer [...] Avec la Traduction des Observations Historiques \& Critiques sur le Mahometisme, mises à la tête de la Version Angloise de M. George Sale, p. 454-455. 
Dans les brouillons des «Imitations du Coran» nous voyons un appel qui correspond entièrement à la traduction citée de Du Ryer: "Begi prorok ot nečestivyh $=\mathrm{O}$ prophète, fuis les impies» ${ }^{62}$.

Ajoutons qu'un motif récurrent dans les lettres de Pouchkine écrites en juin 1824, rappelle fortement la maxime de Saadi ${ }^{63}$. Dans une lettre à Viazemski (24-25 juin 1824), le poète mentionne «le saint commandement du Coran" et le contexte nous permet de supposer qu'il s'agit encore une fois du même conseil: d'éviter les personnes méprisables ${ }^{64}$.

Retournons maintenant à la dernière strophe du «Monument»:

Velen'ju božiju, o muza, bud' poslušna,

Obidy ne strašas', ne trebuja venca,

Hvalu i klevetu priemli ravnodušno,

I ne osporivaj glupca ${ }^{65}$.
A la volonté de Dieu, ô Muse, sois soumise,

Sans craindre l'offense, sans exiger de couronne,

Reçois la louange et la calomnie avec indifférence,

Et ne dispute pas avec le sot.

Maintenant on peut essayer de deviner, pourquoi le dernier précepte de ce quatrain est classé parmi les "volontés de Dieu»: en 1824 Pouchkine croyait qu'il provenait du Coran, tout comme plusieurs autres "pensées sensées» du livre sacré de l'islam. Vers 1836, l'idée de la haute origine de ce "saint commandement" aurait pu perdre ses connotations musulmanes et devenir plus générale.

De toute façon, nous sommes fondés à supposer que le fragment cité de l'"Album d'Onéguine», le début de la troisième "Imitation du Coran" et le vers final du "Monument" remontent aux paraphrases françaises (parfois erronées) du «Gulistan» et du Coran.

\section{Andreï Dobritsyn}

Lausanne

62. A. S. Puškin, Polnoe sobranie sočinenij, t. 2/2, p. 888.

63. Ibid., t. 13, p. 97 et 101. Cf. encore une lettre à Lev Pouchkine écrite en mai 1825, ibid., p. 174.

64. Ibid., p. 100.

65. Ibid., t. 3/1, p. 424. 


\section{BIBLIOGRAPHIE}

Alekseev, M. P., Stihotvorenie Puškina "Ja pamjatnik sebe vozdvig..." . Problemy ego izučenija, Leningrad, 1967.

Almanach des Muses, Paris, 1765-1833.

Billardon de Sauvigny, L.-E., Apologues orientaux, Dédiés à Monseigneur Le Dauphin, Paris, 1764.

Busch, W., Horaz in Russland, München, 1964.

Cardonne, D. D., Mélanges de Littérature Orientale. Traduits de différens Manuscrits Turcs, Arabes \& Persans de la Bibliothèque du Roi, t. 2, Paris, 1770.

Creuzé de Lesser, A., Apologues, Deuxième édition, Paris, 1825.

Desbillons, F.-J., Fables du Père Desbillons Traduites en François, Avec le texte latin Corrigé de nouveau par l'Auteur, t. 1-2, Mannheim, 1779.

Dobritsyn, A. A., "I ne osporivaj glupca..." // Izvestija Rossijskoj Akademii nauk, serija literatury i jazyka, 66,6 (2007), p. 40-44.

Du Ryer, A., L'Alcoran de Mahomet. Traduit de l'Arabe, Par André du Ryer [...] Avec la Traduction des Observations Historiques \& Critiques sur le Mahometisme, mises à la tête de la Version Angloise de M. George Sale, Nouvelle edition, Qu'on a augmentée d'un Discours Preliminaire, extrait du nouvel Ouvrage Anglois de Mr. Porter, t. 2, Amsterdam/Leipzig, 1775.

Dutramblay, A. P., Apologues, Paris, 1822.

Elite de Poësies fugitives, t. 1-5, London, 1769-1770.

Exposition de la Foi Musulmane, traduite du turc de Mohammed Ben Pir-Ali Elberkevi, avec des notes par M. Garcin de Tassy. Suivie de Pend-Nameh, poème de Saadi, traduit du persan, par le même; et du Borda, poème à la louange de Mahomet, traduit de l'arabe, par M. le Baron Sylvestre de Sacy (= Suite du Coran, tome 
troisième, contenant l'Exposition de la Foi Musulmane), Paris/ Leipzig/London, 1828.

Les Flèches d'Apollon ou Nouveau Recueil d'Epigrammes anciennes et modernes [recueilli par E.-J. Chaudon], t. 1-2, London/[Paris], 1787.

[Galland, A.], Les Paroles remarquables, les Bons mots et les Maximes des Orientaux. Traduction de leurs Ouvrages en Arabe, en Persan et en Turc, avec des Remarques, Lyon, 1695.

Galland, A., Les Paroles remarquables, les Bons mots et les maximes des Orientaux. Traduction de leurs ouvrages en arabe, en persan et en turc, avec des remarques, préface d'A. Meddeb, Paris, 1999.

Gleim, J. W. L., Sämmtliche Werke. Erste Originalausgabe aus des Dichters Handschriften durch W. Körte, Bd. V, Halberstadt, 1812.

Huber, M., Choix de Poésies Allemandes, par M. Huber, t. 1-4, Paris, 1766.

Kaštaleva, K. S., «"Podražanija Koranu” Puškina i ih pervoistočnik», in Zapiski kollegii vostokovedov, 1930, t. 5, p. 243-270.

Koebner, R., "Despot and Despotisme: Vicissitude of a Political Term ", Journal of the Warburg and Courtauld Institutes, XIV, 3/4, p. 275-302.

KubačEVA, V. N., “"Vostočnaja povest” v russkoj literature XVIII načala XIX veka», XVIII vek, Sbornik 5, 1962, p. 295-315.

Kurganov, N. [G.], Pismovnik, soderžašcij v sebe nauku rossijskago jazyka, so mnogim prisovokupleniem raznago učebnago i poleznozabavnago vešćslovija, Pjatoe izdanie vnov' vypravlennoe, priumnožennoe i razdelennoe v dve časti, Sankt-Peterburg, 1793.

La Chabeaussière, A.-E.-X., Apologues moraux, imités pour la plupart de Saadi le Persan, [Paris], [entre 1814 et 1820].

[Langlès, L. M.], Contes, Fables et Sentences, Tirés de différens auteurs arabes et persans, avec une analyse du poëme de Ferdoussy, sur les Rois de Perse; par le Traducteur des Instituts politiques et militaires de Tamerlan, Paris, 1778.

Lerner, N. O., "Primečanija. Stihotvorenija 1824 g." in Puškin. Biblioteka velikih pisatelej pod redakciej S. A. Vengerova, SanktPeterburg, 1909, t. 3.

Lestringant, F., "L'Exotisme en France à la Renaissance", in Littérature et exotisme: $X V I^{e}-X V I I I^{e}$ ss., Conférences réunies 
par Dominique de Courcelles, Ecole des Chartes, Paris, 1997, p. 5-16.

Lukman, Fables de Loqman, surnommé le Sage. Edition Arabe, accompagnée d'une Traduction française, et précédée d'une Notice sur ce célèbre Fabuliste, Kaire, An VIII [1799].

Mancini-Nivernois, L. J., Fables, t. 1-2, Paris, 1796.

Massé, H., Essai sur le poète Saadi, suivi d'une bibliographie, Paris, 1919. Modzalevskij, B., Biblioteka A. S. Puškina, Sankt-Petersburg, 1910.

Moura, J.-M., Lire l'Exotisme, Paris, 1992.

Nikolaev, S. I., Ot Kohanovskogo do Mickeviča. Razyskanija po istorii pol'sko-russkih literaturnyh svjazej XVII - pervoj treti XIX v., Sankt-Peterburg, 2004.

Perrin, J.-F., "L'invention d'un genre littéraire au XVIII ${ }^{\mathrm{e}}$ siècle: le conte oriental", Le Conte oriental [Féeries, n'2], Grenoble, 2004/2005, p. 9-28.

Petite Encyclopédie poétique ou Choix de Poésies dans tous les genres, Par une Société de gens de lettres, Paris, 1804, [t. 3] : Epigrammes, madrigaux, épitaphes, inscriptions, pensées, sonnets, rondeaux et triolets, [t. 6]: Fables.

Ротоскі, J., Euvres [t.] 1: Voyage en Turquie et en Egypte. Voyage en Hollande. Voyage dans l'Empire de Maroc, suivi du Voyage de Hafez. Voyage dans quelques parties de la Basse-Saxe, éd. par F. Rosset et D. Triaire, Louvain/Paris/Dudley, MA, 2004.

Puškin, A. S., Polnoe sobranie sočinenij, [Moskva/Leningrad], t. 2/2 (1949): Stihotvorenija 1817-1825, Licejskie stihotvorenija $v$ pozdnejših redakcijah; t. 3/1 (1948): Stihotvorenija 1826-1836, Skazki; t. 6 (1937): Evgenij Onegin; t. 13 (1937): Perepiska 1815-1827.

RAK, V. D., Russkie literaturnye sborniki i periodičeskie izdanija vtoroj poloviny XVIII veka, Sankt-Peterburg, 1998.

Robert, R., «Lecture croisée d'un conte oriental: Pétis de La Croix (Les Mille et Un Jours, 1710), $\mathrm{M}^{1 \mathrm{lle}}$ Falques (Contes du sérail, 1753)", Le Conte oriental [Féeries, n'2], Grenoble, 2004/2005, p. 29-47.

SaAdi, M., Gulistan ou L'Empire des Roses, Composé par Sadi, Prince des Poëtes Turcs et Persans, Traduit par André du Ryer, Paris, 1634. 
—, Gulistan ou l'Empire des Roses, Traité des Mours de Rois, composé par Musladini Saadi, Prince des Poetes Persiens. Traduit du Persan par M.*** [D’Alegre], Paris, 1704.

—, Gulistan ou le Parterre des Roses, par Sadi, traduit du persan sur les meilleurs textes imprimés et manuscrits et accompagné de notes historiques, géographiques et littéraires par Ch. Defrémery, Paris, 1858.

—, Le Boustan ou Verger, Poème persan de Saadi, traduit pour la première fois en français avec une introduction et des notes par A. C. Barbier de Meynard, Paris, 1880.

SAID, Edward, L'Orientalisme: l'Orient créé par l'Occident, traduit de l'américain par Catherine Malamoud, Paris, Seuil, 1997.

Saint-Lambert, J.-F. de, Les Saisons, Poëme, Euvres diverses de l'Auteur des Saisons, Amsterdam, Seconde Partie, 1769.

-, Fables Orientales de M. de S. Lambert, Auteur des Saisons, \&o. Nouvelle édition augmenté de Pensées tirées de Livres Chinois, Arabes, Persans, Turcs, Espagnols, Latins \& François, pour former à la pratique de la Sagesse, \& à la connoissance du cœur humain, Avignon, 1773.

Savary, C., Le Coran, Traduit de l'Arabe, Accompagné de Notes, et précédé d'un Abrégé De la Vie de Mahomet, Tiré des Ecrivains Orientaux les plus estimés, t. 2, Paris, 1783.

—, Le Coran, Traduit de l'Arabe, Accompagné de notes, Précédé d'un abrégé De la Vie de Mahomet, Tiré Des écrivains orientaux les plus estimés. Paris, 1828.

Silvestre de Sacy, A. I., Pend-Namèh, ou Le Livre des Conseils de Férid-

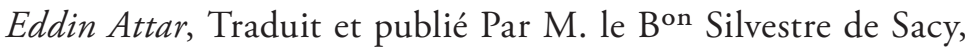
[Paris], 1819.

Le Spectateur ou le Socrate moderne, Où l'on voit un Portrait naïf des Mours de ce Siècle, traduit de l'Anglois, t. 3, Amsterdam, 1718.

Venturi, F., Europe des Lumières. Recherches sur le $18^{\mathrm{e}}$ siècle, Paris, 1971.

Viazemskij, P. A., Polnoe sobranie sočinenij, Izdanie grafa S. D. Šeremeteva, Sankt-Peterburg, 1880, t. 3 : 1808-1827.

—, Stihotvorenija, (L. Ja. Ginzburg, éd.), Leningrad, 1958. 